\title{
ASPIRIN RESISTANCE AFTER CORONARY ARTERY BYPASS GRAFTING
}

\author{
N. Zimmermann, MD, ${ }^{a}$ P. Kienzle, MD, ${ }^{\text {b }}$ A.-A. Weber, MD, ${ }^{b}$ J. Winter, MD, ${ }^{a}$ E. Gams, MD, ${ }^{a}$ K. Schrör, MD, ${ }^{b}$ and \\ T. Hohlfeld, MD, ${ }^{\text {b }}$ Düsseldorf, Germany
}

Aspirin is widely used to reduce graft thrombosis after coronary artery bypass grafting (CABG). ${ }^{1}$ However, aspirin appears to not always be an effective inhibitor of platelet function because considerable individual variations in the antiplatelet effect of aspirin have been reported in patients assigned to $\mathrm{CABG} .^{2}$

From the Institut für Pharmakologie und Klinische Pharmakologie ${ }^{\mathrm{b}}$ and the Klinik für Thorax- und Kardiovaskuläre Chirurgie, ${ }^{a}$ Heinrich Heine-Universität, Düsseldorf, Germany.

J Thorac Cardiovasc Surg 2001;121:982-4

Copyright (C) 2001 by The American Association for Thoracic Surgery

$0022-5223 / 2001 \$ 35.00+0 \quad \mathbf{1 2 / 5 4 / 1 1 1 4 1 6}$

doi: $10.1067 / \mathrm{mtc} .2001 .111416$
The antiplatelet effect of aspirin may be compromised by an enhanced platelet turnover, generating an increased fraction of platelets that is able to form thromboxane within the dosing intervals (usually 1 day). This may be of particular relevance for patients undergoing CABG after cardiopulmonary bypass, when postoperative platelet turnover is increased.

The present work examines whether the antiplatelet effect of aspirin, which is based on the inhibition of platelet thromboxane production, may be impaired by an increased platelet turnover. Determination of thromboxane in collagen-stimulated platelet-rich plasma after CABG showed a remarkable enhancement of the capacity for thromboxane formation, despite the fact that aspirin was administered at an antiplatelet dose (100 mg once daily) that largely suppressed thromboxane synthesis in healthy control subjects. 

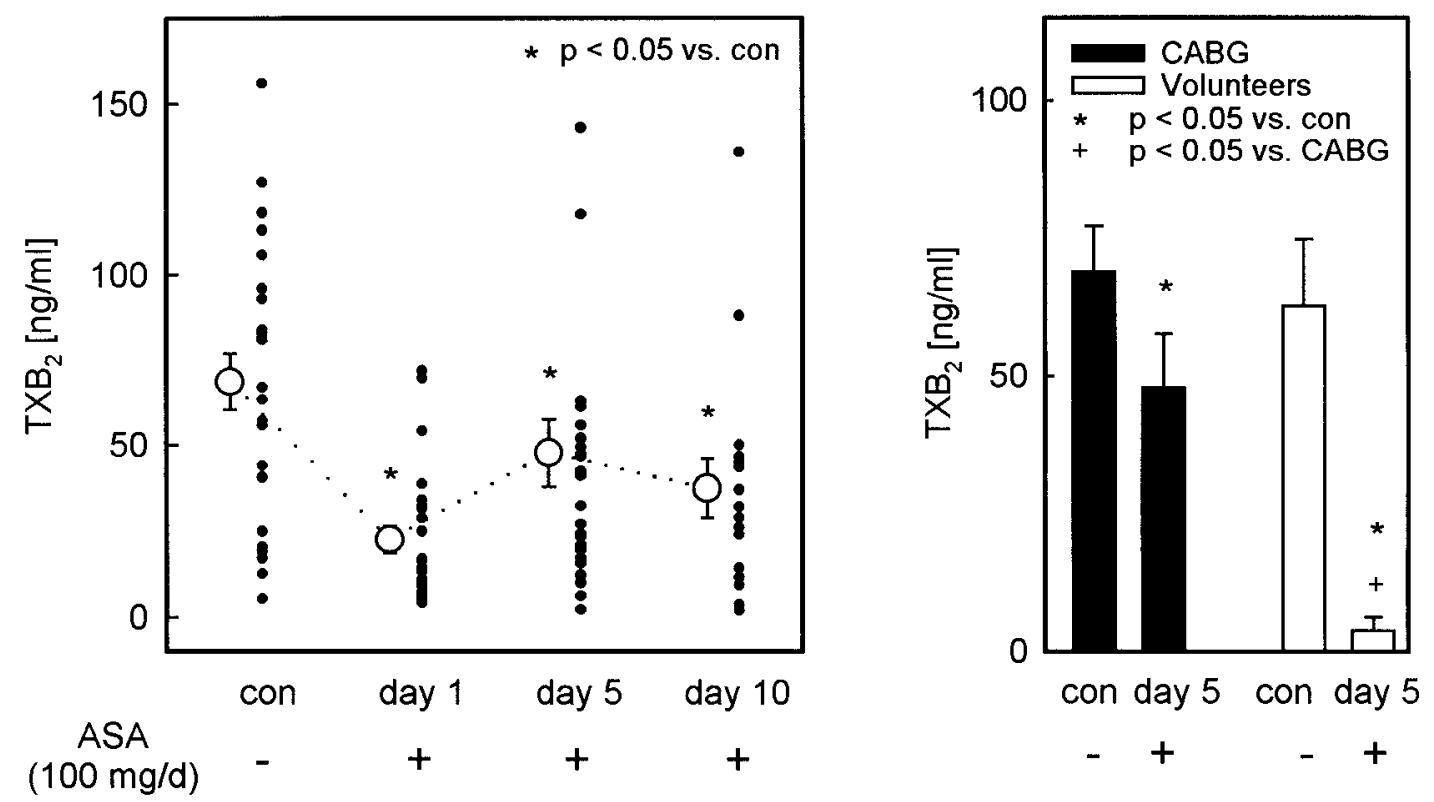

Fig 1. Left, Concentrations of immunoreactive thromboxane (TX) $\mathrm{B}_{2}$ measured ex vivo after collagen-induced (1 $\mu \mathrm{g} / \mathrm{mL}$ collagen) platelet activation. Measurements were obtained before $\mathrm{CABG}$ (con) and at days 1, 5, and 10 after CABG. Aspirin (ASA) treatment (100 mg/d) started at day 1 after CABG. Data are mean values \pm SEM (open circles) and individual measurements (filled circles). Right, Platelet thromboxane (TX) formation before (con) and after aspirin $(A S A)$ administration for 5 days in patients undergoing CABG (filled bars) in comparison with healthy control subjects (open bars).

\section{Methods}

Subjects and drug administration. This study was conducted in agreement with the Declaration of Helsinki and was approved by the institutional ethics committee. Twenty-four consecutive patients were included who had stable coronary artery disease and underwent an elective CABG procedure (2- to 3-vessel disease). Informed written consent was obtained from each patient. Previous aspirin treatment was terminated at least 7 days before CABG. Tablets (Aspirin 100 protect; Bayer AG, Leverkusen, Germany) containing $100 \mathrm{mg}$ of aspirin were administered every morning, starting on day 1 after the operation. Compliance was monitored by means of tablet counts. For comparison, 5 healthy male volunteers (age range, 27-45 years) received aspirin treatment (100 mg once daily) over a duration of 5 days.

Measurement of platelet function. Venous blood $(10 \mathrm{~mL})$ was collected in the morning (before aspirin ingestion) from patients 1 day before and at days 1, 5, and 10 after CABG and from volunteers immediately before and at day 5 of aspirin treatment. Blood was drawn from the antecubital vein into citrated (1:9) Vacutainer systems (Becton Dickinson, Franklin Lakes, NJ). Platelet counts were determined by means of automated counting. Immediately thereafter, platelet-rich plasma was prepared, and collagen-induced (1 $\mu \mathrm{g} / \mathrm{mL}$ collagen) aggregation and thromboxane formation were determined, as previously described. ${ }^{3}$
Statistics. Data are mean values \pm SEM. Statistical analyses were performed with the Student $t$ test and analysis of variance for repeated measures, as required.

Results. CABG caused an early decrease in platelet counts from $221 \pm 8 \times 10^{3} / \mu \mathrm{L}$ (before $\mathrm{CABG}$ ) to $160 \pm 9 \times 10^{3} / \mu \mathrm{L}$ (day 1 after CABG, $P<.05$ ). Thereafter, platelet counts increased to $266 \pm 14 \times 10^{3} / \mu \mathrm{L}$ at day 5 and $454 \pm 31 \times$ $10^{3} / \mu \mathrm{L}$ at day 10 , indicating a substantial recovery of platelet counts, which, at days 5 and 10 , significantly exceeded the control values obtained before CABG $(P<.05)$.

Before $\mathrm{CABG}, 1 \mu \mathrm{g} / \mathrm{mL}$ collagen caused a considerable platelet release of thromboxane (Fig 1). This was moderately reduced at day 1 after $\mathrm{CABG}$, as shown by a decay of thromboxane to $33 \%$ of the control value $(P<.05)$. Nevertheless, this inhibition was incomplete, and thromboxane formation remained high at days 5 and 10 after CABG, corresponding to $70 \%$ and $54 \%$ of the control values before the operation. By contrast, the same dose of aspirin $(100 \mathrm{mg} / \mathrm{d})$ administered to healthy volunteers for 5 days largely suppressed thromboxane to $6 \%$ of the control value $(P<.05$, Fig 1$)$.

This marginal inhibition of platelet thromboxane formation by aspirin in patients undergoing CABG did not result in a significant inhibition of platelet function, as shown by collagen-induced platelet aggregation. At days 1, 5, and 10 after CABG, platelet aggregation was $92 \% \pm 8 \%, 103 \% \pm 7 \%$, and $91 \% \pm 5 \%$ of the control value before the operation $(100 \%)$. 
By contrast, aspirin ( $100 \mathrm{mg} / \mathrm{d}$ for 5 days) significantly inhibited platelet aggregation in healthy subjects to $33 \% \pm 16 \%$ of the pretreatment value $(P<.05)$.

Discussion. The major finding of this study is that $100 \mathrm{mg}$ of aspirin, a dose known to be effective for the early antithrombotic treatment after $\mathrm{CABG},{ }^{4,5}$ did not inhibit platelet collagen-induced ( $1 \mu \mathrm{g} / \mathrm{mL}$ collagen) aggregation and only incompletely inhibited platelet thromboxane synthesis in patients subjected to CABG. The same dose and a comparable duration of treatment (5 days) significantly inhibited aggregation and almost completely abrogated thromboxane formation of platelets from healthy subjects. Hence, the early postoperative period after CABG is characterized by a remarkable resistance against the antiplatelet effect of aspirin.

It is important to recall, in this context, that cardiac operations with cardiopulmonary bypass, including the majority of CABG procedures, activate and partially deplete circulating platelets. ${ }^{6}$ In the present study this resulted in a fall of the platelet count by about $25 \%$ at the first day after CABG. Thereafter, platelet counts rapidly recovered. At day 10 after CABG, platelet counts rose to more than $100 \%$ above the value before CABG. Assuming a median platelet lifetime of 8 to 10 days, platelet regeneration was therefore augmented at least 2-fold within the first 10 days after the operation.

Hence, immediately before ingestion of the daily aspirin dose, a significant amount (ie, $\geq 10 \%$ ) of circulating platelets were probably competent for thromboxane formation. Given that (1) platelet thromboxane synthesis needs to be blocked to less than $10 \%$ to achieve efficient platelet inhibition ${ }^{7}$ and (2) the plasma half-life of aspirin is short (ie, $<30$ minutes), ${ }^{8}$ circadian inhibition of platelet function may be incomplete. In fact, the present study clearly shows that aspirin did not sufficiently block thromboxane formation to allow for significant platelet inhibition. One may argue that the method of platelet aggregation measurements might not have been sensitive enough to detect a moderate inhibitory effect of aspirin. This, however, is unlikely because a comparable treatment of healthy subjects significantly inhibited platelet aggregation in this and earlier studies. ${ }^{9}$

Nevertheless, it is possible that additional causes of platelet resistance against aspirin may be involved. For example, an alternative aspirin-insensitive pathway for platelet thromboxane formation, such as induction of the inducible isoform of cyclooxygenase, may contribute. ${ }^{10} \mathrm{~A}$ follow-up trial has been started to examine the pharmacodynamic properties and adequate dosing intervals of aspirin after CABG.

The present study provides some implications for the use of aspirin to reduce bypass thrombosis after CABG. First, conventional doses of aspirin, such as $100 \mathrm{mg} / \mathrm{d}$, appear ineffective to suppress platelet thromboxane formation in the majority of patients. Second, at least one reason for this aspirin resistance seems to be a considerably increased recruitment of new platelets. The current results suggest that the problem may be overcome by administration of aspirin in more than one daily dose, which should prevent a critical circadian increase of the new thromboxane-forming platelet fraction. Third, monitoring of the antiplatelet effect of aspirin after CABG and potentially other states of enhanced platelet regeneration appears worth considering. This would allow for an optimized antiplatelet regimen in those patients who experience aspirin resistance, including the use of alternative antiplatelet drugs.

We thank Irmhild Rüter, Kirsten Bartkowski, and Susanne Heck for technical assistance and Erika Lohmann for secretarial work.

Received for publication May 12, 2000; accepted for publication Sept 4, 2000.

Address for reprints: Thomas Hohlfeld, MD, Institut für Pharmakologie und Klinische Pharmakologie, Heinrich Heine-Universität, Moorenstraße 5, 40225 Düsseldorf, Germany (E-mail: hohlfeld@uni-duesseldorf.de).

\section{REFERENCES}

1. Motwani JG, Topol EJ. Aortocoronary saphenous vein graft disease. Pathogenesis, predisposition, and prevention. Circulation 1998;97:916-31

2. Buchanan MR, Brister SJ. Individual variation in the effects of ASA on platelet function: implications for the use of ASA clinically. Can J Cardiol 1995;11:221-7.

3. Weber A-A, Liesener S, Hohlfeld T, Schrör K. $40 \mathrm{mg}$ of aspirin are not sufficient to inhibit platelet function under conditions of limited compliance. Thromb Res 2000;97:365-7.

4. Reilly IA, FitzGerald GA. Aspirin in cardiovascular disease. Drugs 1988;35:154-76.

5. Stein PD, Dalen JE, Goldman S, Schwartz L, Theroux P, Turpie AG. Antithrombotic therapy in patients with saphenous vein and internal mammary artery bypass grafts. Chest 1995;108:424S$30 \mathrm{~S}$.

6. Hyde JA, Chinn JA, Graham TR. Platelets and cardiopulmonary bypass. Perfusion 1998;13:389-407.

7. FitzGerald GA, Reilly IA, Pedersen AK. The biochemical pharmacology of thromboxane synthase inhibition in man. Circulation 1985;72:1194-201.

8. Benedek IH, Joshi AS, Pieniaszek HJ, King SYP, Kornhauser DM. Variability in the pharmacokinetics and pharmacodynamics of low dose aspirin in healthy male volunteers. J Clin Pharmacol 1995;35:1181-6.

9. Schrör K. Aspirin and platelets: the antiplatelet action of aspirin and its role in thrombosis treatment and prophylaxis. Semin Thromb Hemost 1997;23:349-56.

10. Weber A-A, Zimmermann K, Meyer-Kirchrath J, Schrör K. Cyclooxygenase-2 in human platelets as a possible factor in aspirin resistance. Lancet 1999;353:900. 